\title{
Effect of Fluid Intake on Hydration Status and Skin Barrier Characteristics in Geriatric Patients: An Explorative Study
}

\author{
Merve Akdeniz ${ }^{a}$ Heiner Boeing ${ }^{b}$ Ursula Müller-Werdan ${ }^{c}$ Volkan Aykac ${ }^{c}$ \\ Annika Steffen $^{\mathrm{b}}$ Mareike Schell ${ }^{\mathrm{b}} \quad$ Ulrike Blume-Peytavi $^{\mathrm{a}}$ Jan Kottner ${ }^{\mathrm{a}}$ \\ aClinical Research Center for Hair and Skin Science, Department of Dermatology and Allergy, Charité - \\ Universitätsmedizin Berlin, Berlin, Germany; ${ }^{b}$ German Institute of Human Nutrition Potsdam-Rehbrücke (DIfE) \\ Department of Epidemiology, Berlin, Germany; ' ${ }^{\complement}$ Evangelic Geriatric Center, Charité - Universitätsmedizin Berlin, \\ Berlin, Germany
}

\section{Keywords}

Transepidermal water loss $\cdot \mathrm{pH} \cdot$ Dehydration $\cdot$ Skin physiology $\cdot$ Drinking $\cdot$ Geriatrics

\begin{abstract}
Background/Aim: Inadequate fluid intake is assumed to be a trigger of water-loss dehydration, which is a major health risk in aged and geriatric populations. Thus, there is a need to search for easy to use diagnostic tests to identify dehydration. Our overall aim was to investigate whether skin barrier parameters could be used for predicting fluid intake and/or hydration status in geriatric patients. Methods: An explorative observational comparative study was conducted in a geriatric hospital including patients aged 65 years and older. We measured 3-day fluid intake, skin barrier parameters, Overall Dry Skin Score, serum osmolality, cognitive and functional health, and medications. Results: Forty patients were included (mean age 78.45 years and 65\% women) with a mean fluid intake of $1,747 \mathrm{~mL} /$ day. $20 \%$ of the patients were dehydrated and $22.5 \%$ had an impending dehydration according to serum osmolality. Multivariate analysis suggested that skin surface $\mathrm{pH}$ and epidermal hydration at the face were associated with fluid intake. Serum osmolality was associated with epidermal hydration at the leg and skin surface
\end{abstract}

\section{KARGER}

๑ 2018 S. Karger AG, Basel

E-Mail karger@karger.com

www.karger.com/spp
$\mathrm{pH}$ at the face. Fluid intake was not correlated with serum osmolality. Diuretics were associated with high serum osmolality. Conclusions: Approximately half of the patients were diagnosed as being dehydrated according to osmolality, which is the current reference standard. However, there was no association with fluid intake, questioning the clinical relevance of this measure. Results indicate that single skin barrier parameters are poor markers for fluid intake or osmolality. Epidermal hydration might play a role but most probably in combination with other tests.

ㄷ) 2018 S. Karger AG, Basel

\section{Introduction}

Water is the main component of the human body, and it is essential for life. Nearly all biological processes including growth, metabolism, cellular homeostasis, movement, and signaling require an adequate aqueous environment to function properly. During ageing, the proportion of body water reduces from approximately $75 \%$ in infants to $55 \%$ in the elderly [1]. Dehydration is a complex condition, which can be due to water-loss dehydration or salt-loss dehydration, resulting in reduction in total body water [2]. 
In healthy adults, restricted fluid intake increases the serum osmolality, activates thirst and releases vasopressin (antidiuretic hormone), resulting in decreased renal water loss and stimulation of fluid intake. Due to physiological changes associated with aging (e.g., decreased thirst responses, changes in renal function, altered vasopressin levels, polypharmacy, diuretics, decreased cognitive function) older persons have an increased risk of dehydration. Especially in geriatric patients, water-loss dehydration is associated with poor health outcomes, including falls, fractures, constipation, confusion, drug toxicity, and death [3]. Available studies especially suggest a relationship between hydration status and cognitive performance $[4,5]$. Therefore, interventions to improve fluid intake in aged care receivers and geriatric patients are considered of utmost importance [6]. However, in clinical practice identifying geriatric patients at risk or with (early) signs of dehydration is challenging. Various clinical tests and methods have been proposed to diagnose dehydration (e.g., "skin turgor test," expressing fatigue, missing drinks between meals, dry mouth, and tongue furrows), but a recent Cochrane review indicated that the evidence supporting the diagnostic accuracy of these tests is extremely weak [3].

Serum osmolality is regarded as the diagnostic reference standard to indicate water-loss dehydration. However, the wide and routine determination of serum osmolality in geriatric and long-term care settings is not feasible. Therefore, there is a need for simple noninvasive but accurate diagnostic procedures to identify geriatric patients at risk for or with dehydration. Early identification, prevention, and targeted treatment of dehydration would improve the quality of life and well-being and prevent adverse events like emergency hospital admissions.

Empirical evidence suggests relationships between fluid intake and skin properties $[7,8]$. An additional intake of water seems to influence skin barrier parameters like stratum corneum hydration (SCH), in particular in individuals with lower prior water consumption $[9,10]$. Associations between fluid intake and skin surface $\mathrm{pH}$ have also been described [11]. Such associations might also exist in aged and geriatric patients but have never been investigated so far. Adequate fluid intake is commonly believed to play an important role to ensure an adequate skin hydration. However, the scientific evidence about the association between fluid intake and skin barrier properties seems to be sparse [12]. Therefore, the overall aim of this study was to investigate whether skin barrier parameters are associated with fluid intake and hydration status in geriatric patients.

\section{Methods}

\section{Study Design and Setting}

An explorative observational comparative study was conducted in the Evangelic Geriatric Center of Berlin, Germany, between April and June 2016. Within this period, geriatric patients were recruited and followed up. The study protocol and the informed consent forms and procedures were approved by the Ethics Committee of the Charité - Universitätsmedizin Berlin. Participation was voluntary. Patients were only included after having signed the informed consent and the study conduct followed the principles of the Declaration of Helsinki.

\section{Participants}

Due to the explorative nature of the study, a formal sample size determination was not conducted. We regarded $n=40$ patients as sufficient to detect possible differences and/or associations between variables. Patients had to meet the following inclusion criteria: age 65+ years old, written informed consent (or by legal representative), and assumed ability that skin measurements can be performed. Following exclusion criteria were applied: Patients at the end of life (dying persons), any dermatological condition or skin affection, e.g. acute weeping, excoriated or inflammatory dermatitis, or skin treatment which may interfere with the study assessments at the discretion of the investigator, any unstable acute or chronic pathology or condition that may interfere with the study conduct at the discretion of the investigator, any use of topical drugs on the investigational areas 2 weeks prior to inclusion, systemic application of corticosteroid. Potentially eligible patients were approached directly by study assistants and investigators. Written informed consent was obtained from the patients directly or by their legal representatives.

\section{Variables and Data Collection}

Demographics and variables characterizing medical and functional conditions were measured. The Mini-Mental State Examination (MMSE) was used to measure the mental status on a scale from 0 to 30 [13]. A score of 23 or lower was regarded as indicative of cognitive impairment. The Barthel Index was used to assess the dependency in the activities of daily living [14]. The 30-item Geriatric Depression Scale was used to measure depression. All these scores were extracted from the medical records including medications and documented signs of dehydration. Fluid intake (e.g., drinks) was recorded by nurses and/or by the patients themselves, and the volume of intravenous medication was obtained from the clinical records for 3 consecutive days. At day 3, blood samples were drawn, and serum osmolality was measured. Hydration status was classified as normally hydrated ( 275 to $<295 \mathrm{mOsm} / \mathrm{kg}$ ), impending dehydration ( 295 to $\leq 300 \mathrm{mOsm} / \mathrm{kg}$ ), and current dehydration (>300 mOsm/kg) [2]. The clinical assessment of the presence or severity of skin dryness (xerosis) was performed by a physician using the Overall Dry Skin Score (ODS) on a five-point scale from 0 to 4 which was separately assessed for arms, face, trunk and legs [15]. The skin barrier function was characterized by four parameters: transepidermal water loss (TEWL) in $\mathrm{g} / \mathrm{m}^{2} / \mathrm{h}$, skin surface $\mathrm{pH}$, epidermal hydration in percentage of local tissue water, $\mathrm{SCH}$ in arbitrary units on the three skin areas: forehead, outer forearm, and outer lower leg. 
TEWL indicates the passive diffusion of water molecules through the stratum corneum. Measurements were performed with the Tewameter TM 300 (Courage and Khazaka Electronic $\mathrm{GmbH}$, Cologne, Germany) in duplicate and the arithmetic mean was used. Measurements were performed according to standard operating procedures based on international guidelines [16], and the reliability and validity of TEWL measurements have been demonstrated [17].

SCH and epidermal hydration indicate the proportion of water molecules within the respective skin layers. The Corneometer CM 825 (Courage and Khazaka Electronic) was used to measure SCH. The probe was held vertically and placed on the skin with a light constant pressure 3 times subsequently per skin area. The same procedure was repeated and the arithmetic mean of these values was recorded. The reliability and validity of SCH measurements have been demonstrated [18]. Epidermal hydration was measured using the Moisture MeterEpiD (Delfin Technologies). The probe was held vertically and placed on the skin with a constant pressure 2 times subsequently per skin area. The arithmetic mean of these values was recorded.

The skin surface $\mathrm{pH}$ was measured with a glass electrode attached to the Skin-pH-Meter PH905 (Courage and Khazaka Electronic). The assessment of skin surface $\mathrm{pH}$ was conducted according to international guidelines [19]. Skin surface temperature $\left({ }^{\circ} \mathrm{C}\right)$ was measured with the skin-Thermometer ST 500 (Courage and Khazaka Electronic).

All instrumental skin measurements were performed in the patient rooms in the morning between 08:00 and 10:00 a.m. while patients were resting and before any skin products were applied. Relative humidity and the room temperature were recorded. The patients were instructed to refrain from washing or applying any topical products to the test sites for at least $12 \mathrm{~h}$ prior to the measurements.

\section{Statistical Methods}

In a first step, sample characteristics were described using mean and spread parameters and proportions. Next, bivariate associations were described in a correlation matrix using Spearman correlation coefficients. A correlation of 0.2 or higher or -0.2 or lower was regarded as minimum strengths to indicate possible association. The sample was divided into tertiles according to fluid intake and the number of patients with normal $(<295 \mathrm{mOsm} / \mathrm{kg})$ and high ( $\geq 295 \mathrm{mOsm} / \mathrm{kg}$ ) osmolality displayed (see Table 2 ).

Finally, multiple linear regression analysis with LASSO (Least Absolute Shrinkage and Selection Operator) selection was applied to identify those variables (from the whole potential set of medical variables and skin parameters) that were most strongly related to fluid intake and osmolality. Importantly, obtained parameter estimates in this model cannot be interpreted as such because we reversed dependent and independent variables by modelling fluid intake/osmolality as single dependent variable and the various skin parameters as independent variables. This was done because it was not possible to simultaneously model numerous skin parameters as dependent variables with only one independent variable (fluid intake/osmolality predicting skin parameters). However, the approach enabled us to identify those skin parameters that are most likely associated with fluid intake/osmolality. All $p$ values were two-sided. All analyses were performed in SAS (version 9.4, Enterprise Guide 4.3, SAS Institute Inc., Cary, NC, USA).

Fluid Intake and Skin Barrier

\section{Results}

\section{Participants and Descriptive Data}

The demographic and other characteristics are shown in Table 1. Mean age was 79 (SD 6.7) years and 65\% were female. Mean BMI was 26.2 (SD 4.3). Mean MMSE score was 24. Mean BI was 45 and mean Geriatric Depression Scale score was 3. Mean fluid intake was $1,747 \mathrm{~mL}$ per day, and clinical signs of dehydration were documented for 5 patients (12.5\%). The mean number of prescribed medications was 10.6 (SD 3.6), and $67.5 \%$ of the complete listing of medications was diuretics.

Mean serum osmolality was 294 (SD 9.9) $\mathrm{mOsm} / \mathrm{kg}$; $23 \%$ had impending dehydration, and $20 \%$ had current dehydration according to the thresholds. The highest mean ODS was 1.7 recorded on the legs, and the lowest was 0.3 on the face. Facial skin also showed highest TEWL, $\mathrm{SCH}$, and epidermal hydration values. Skin surface $\mathrm{pH}$ values varied slightly between 5.0 and 5.3.

\section{Associations}

The tertiles of fluid intake are shown in Table 2. According to this, fluid intake seems to be unrelated to serum osmolality, but patients with diuretics nearly always were dehydrated. The mean number of medications was unrelated to the hydration status. Results of the bivariate associations are shown in a correlation matrix in the online supplementary file 1 (for all suppl. material, see www. karger.com/doi/10.1159/000487403). Correlation coefficients higher than 0.2 or lower than -0.2 are highlighted. According to this matrix, the fluid intake showed the highest positive association with epidermal hydration at the forehead $\left(r_{\mathrm{s}}=0.33\right)$. Serum osmolality was positively associated with the ODS at the face $\left(r_{\mathrm{s}}=0.31\right)$ and with serum natrium $\left(r_{\mathrm{s}}=0.51\right)$. There were negative relationships with epidermal hydration at the leg $\left(r_{\mathrm{s}}=-0.31\right)$, skin surface $\mathrm{pH}$ at the face $\left(r_{\mathrm{s}}=-0.29\right)$, sex $\left(r_{\mathrm{s}}=-0.40\right)$, and $\operatorname{MMSE}\left(r_{\mathrm{s}}=-0.35\right)$.

A number of other bivariate associations were observed. For instance, a negative correlation was observed between ODS and sex $(r=-0.47)$. Men tended to have a dryer facial skin than women. A negative correlation was observed between MMSE and the use of diuretics $\left(r_{s}=-0.43\right)$ and also between serum osmolality and MMSE $\left(r_{s}=-0.35\right)$. There was no association between fluid intake and osmolality.

Highest associations overall were observed between intraindividual skin barrier parameters. For instance, skin surface $\mathrm{pH}$ at the arm was strongly associated with the $\mathrm{pH}$ at the leg $\left(r_{\mathrm{s}}=0.64\right)$ and face $\left(r_{\mathrm{s}}=0.70\right)$. Similarly, the correlation between $\mathrm{SCH}$ at the arm and the face was 
Table 1. Characteristics of study participants $(n=40)$

\begin{tabular}{|c|c|c|c|}
\hline Age, years & $78.5(6.7)$ & $78.0(75.0 ; 84.5)$ & - \\
\hline Female, $n(\%)$ & $26(65.0)$ & & - \\
\hline Body mass index & $26.2(4.3)$ & $25.5(23.5 ; 29.1)$ & 1 \\
\hline Barthel index & $45.3(21.3)$ & $45.0(30.0 ; 60.0)$ & 1 \\
\hline Mini-Mental State Examination & $24.1(4.9)$ & $25.00(22.0 ; 29.0)$ & 3 \\
\hline Clinical signs of water-loss dehydration, $n(\%)$ & $5(12.5)$ & & - \\
\hline Medications, $n$ & $10.6(3.6)$ & & - \\
\hline Diuretics, $n(\%)$ & $27(67.5)$ & & - \\
\hline Serum osmolality, $\mathrm{mOsm} / \mathrm{kg}$ & $293.5(9.9)$ & $293.0(288.0 ; 298.5)$ & - \\
\hline$<295, n(\%)$ & & $23(57.5)$ & \\
\hline Face & $0.3(0.6)$ & $0.0(0.0 ; 0.0)$ & - \\
\hline Arms & $1.4(0.9)$ & $1.0(1.0 ; 2.0)$ & - \\
\hline Legs & $1.7(1.1)$ & $2.0(1.0 ; 2.0)$ & - \\
\hline Transepidermal water loss, $\mathrm{g} / \mathrm{m}^{2} / \mathrm{h}$ & & & - \\
\hline Face & $14.3(5.2)$ & & \\
\hline Arms & $9.4(5.3)$ & & \\
\hline Legs & $6.9(2.7)$ & & \\
\hline Stratum corneum hydration, AU & & & - \\
\hline Face & $54.0(15.9)$ & & \\
\hline Arms & $38.9(10.0)$ & & \\
\hline Legs & $31.4(10.6)$ & & \\
\hline
\end{tabular}

Table 2. Medication by tertiles of fluid intake per day and hydration status ( $\mathrm{mOsm} / \mathrm{kg})(n=40)$

\begin{tabular}{|c|c|c|c|c|c|c|}
\hline Hydration status & $<295$ & $\geq 295$ & $<295$ & $\geq 295$ & $<295$ & $\geq 295$ \\
\hline Medications, mean $n(95 \% \mathrm{CI})$ & $11.1(8.5 ; 13.8)$ & $11.9(9.0 ; 14.7)$ & $7.7(5.1 ; 10.4)$ & $9.8(6.7 ; 12.9)$ & $10.7(8.3 ; 13.0)$ & $13.3(9.5 ; 17.0)$ \\
\hline Use of diuretics, $n$ (\%) & $6(85.71)$ & $6(85.71)$ & $1(14.29)$ & $6(100.00)$ & $4(44.44)$ & $4(100.00)$ \\
\hline
\end{tabular}


Table 3. Variable retained in LASSO penalized regression for fluid intake (adj. $R^{2}=0.13$ )

\begin{tabular}{lrlrl}
\hline Variable & $\begin{array}{l}\text { Parameter } \\
\text { estimate }\end{array}$ & $\begin{array}{l}\text { 95\% confidence } \\
\text { limits }\end{array}$ & $p$ value \\
\hline Intercept & 3.08 & -23.39 & 29.54 & 0.81 \\
Age & -0.05 & -0.23 & 0.13 & 0.56 \\
ODS face & 1.09 & -1.11 & 3.29 & 0.32 \\
TEWL head & 0.02 & -0.32 & 0.36 & 0.91 \\
TEWL arm & 0.28 & -0.06 & 0.62 & 0.10 \\
SCH leg & 0.04 & -0.09 & 0.18 & 0.51 \\
SCH arm & -0.03 & -0.18 & 0.11 & 0.65 \\
EH head & 0.14 & -0.03 & 0.31 & 0.10 \\
pH head & 3.70 & 0.08 & 7.32 & 0.05 \\
pH arm & -1.43 & -6.00 & 3.13 & 0.53 \\
pH leg & -0.77 & -4.16 & 2.61 & 0.64 \\
\hline
\end{tabular}

ODS, Overall Dry Skin Score; TEWL, transepidermal water loss; SCH, stratum corneum hydration; EH, epidermal hydration.

Table 4. Results of the Lasso regression model for serum osmolality $\left(\right.$ adj. $\left.R^{2}=0.38\right)$

\begin{tabular}{lcrrr}
\hline Variable & $\begin{array}{l}\text { Parameter } \\
\text { estimate }\end{array}$ & \multicolumn{2}{l}{$\begin{array}{l}\text { 95\% confidence } \\
\text { limits }\end{array}$} & $p$ value \\
\hline Intercept & 337.00 & 304.79391 & 369.21 & $<0.0001$ \\
Sex & -4.44 & -10.39615 & 1.51 & 0.1382 \\
Diuretics & 3.18 & -2.98784 & 9.35 & 0.3012 \\
Medications & 0.58 & -0.29987 & 1.45 & 0.1896 \\
ODS arm & 1.41 & -2.04612 & 4.87 & 0.4110 \\
ODS face & 4.12 & -1.04637 & 9.28 & 0.1140 \\
SCH leg & 0.23 & -0.08401 & 0.55 & 0.1438 \\
EH leg & -0.52 & -0.83820 & -0.20 & 0.0023 \\
pH leg & -5.94 & -11.80032 & -0.08 & 0.0472 \\
\hline
\end{tabular}

ODS, Overall Dry Skin Score; SCH, stratum corneum hydration; $\mathrm{EH}$, epidermal hydration.

positive $\left(r_{\mathrm{s}}=0.61\right)$. A positive association was detected between $\mathrm{SCH}$ at the leg and at the $\operatorname{arm}\left(r_{\mathrm{s}}=0.50\right)$. A positive correlation was observed between ODS at the leg and ODS at the arm $\left(r_{\mathrm{s}}=0.55\right)$ A similar association was observed between $\mathrm{SCH}$ at the leg and ODS at the leg $(r=$ $-0.49)$. An association was observed between $\mathrm{SCH}$ at the leg and epidermal hydration at the leg $(r=0.57)$.

\section{Multivariate Analyses}

In total, 10 variables appeared to be relevant for predicting fluid intake $\left(R^{2}=0.13\right)$ (Table 3). Except age, all selected variables were related to skin properties. Skin surface $\mathrm{pH}$ at the face showed the strongest association with fluid intake. Eight predictors were selected for the dependent variable osmolality $\left(R^{2}=0.38\right)$ (Table 4$)$. Higher osmolality was significantly associated with epidermal hydration and skin surface $\mathrm{pH}$ at the leg.

\section{Discussion}

The overall aim of this study was to investigate whether skin barrier parameters are associated with fluid intake and hydration status in geriatric patients. In terms of gender and BI, the sample characteristics were comparable to typical geriatric and long-term care populations in Germany $[20,21]$. The measured median fluid intake of approximately $1,700 \mathrm{~mL}$ per day may be considered as appropriate for this population [22] and the interquartile range from 1,500 to $1,900 \mathrm{~mL} /$ day indicates that variations were small. The measured skin barrier parameters in the sample are similar to previous research in aged individuals $[21,23,24]$. From face to arm to leg there was a decrease in $\mathrm{SCH}$ and TEWL, indicating increasing skin dryness which is supported by increasing clinical dryness scores. Xerosis is a common dermatological challenge in the elderly [25]. Especially in aged and geriatric populations, distal extremities are affected by dry skin [26].

Fluid intake was associated with epidermal hydration of the face and leg. This may indicate that increased water intake in geriatric patients may be related to the water content in the epidermal cells beneath the stratum corneum. This empirical finding is supported by Palma et al. $[9,10,27]$ who showed increased epidermal hydration after increased water consumption in younger populations. It is well known that increased fluid intake increases the water content in the dermis because the water accumulates in this layer [28]. The glycosaminoglycan polymer hyaluronan is a highly hydrophilic component of the extracellular matrix, which occurs in both dermis and epidermis and provides the skin hydration and turgor [29]. Increased water content in the dermis might also increase the water content at the epidermal level.

No other bivariate correlations have been observed between fluid intake and skin barrier parameters, whereas in the multivariate analysis, fluid intake was positively associated with $\mathrm{pH}$ on the face. We are aware of previous studies showing statistical significant associations between fluid intake and $\mathrm{pH}[11,30]$ and SCH $[9,10,27]$. However, the underlying biological mechanisms are unclear and younger healthy populations are not comparable with geriatric patients. 
In the multivariate analysis, age and 9 skin-related variables were identified to explain fluid intake. This indicates that these skin properties in combination are directly or indirectly associated with fluid intake. However, this model was able to explain less than $13 \%$ of variance, indicating that that there are many more factors predicting fluid intake which were not considered in this study.

Mean serum osmolality was $294 \mathrm{mOsm} / \mathrm{kg}$, but according to the applied classification 9 patients showed impeding and 8 patients showed water-loss dehydration. Negative bivariate associations were observed between serum osmolality and epidermal hydration of the leg and $\mathrm{pH}$ of the leg. Biological explanations are challenging. Effective solutes in plasma, which are impermeable to cell membranes, influence cell volume via their osmotic force on cells. Abnormally raised osmolarity implies cell dehydration as intracellular fluid moves to extracellular space which results in shrinking of cells. This shrinkage might occur in epidermal cells.

Another possible explanation might be the anatomical location. It is well known that the most common site for xerosis cutis in the elderly is the lower leg, with almost three-quarters of all xerosis cases being diagnosed at this location [31]. We are aware of one study of Palma et al. [27] that evaluated the effect of an increased dietary intake of water on skin hydration. They reported smaller hydration increases at the zygomatic area, where cosmetic products are usually applied. Hydration changes might be more noticeable in less dehydrated areas. In the multivariate model, 8 predictors were selected but only 5 were related to skin properties, and this model explained nearly $40 \%$ of variance. Interestingly, lower epidermal hydration at the leg was significantly associated with higher osmolality.

\section{Other Associations}

An important finding of our study was that there was no association between fluid intake and osmolality. This indicates that other factors (e.g., hormonal changes during ageing, chronic diseases) might play a more important role for controlling serum osmolality than the fluid intake itself. The adjustment of serum osmolality in geriatric patients may be regarded as a rather long-term process. This leads to the question whether serum osmolality is actually the best reference standard for measuring dehydration and whether the used cut-offs are truly useful for clinical decision-making [3]. The question is whether increased serum osmolality should automatically lead to increased fluid intake in geriatric patients. Guidelines and clinical practice recommendations widely propose to ensure that patient's hydration is adequate $[22,32]$. How- ever, the question of what is adequate in geriatric patients is not easy to answer. At the same time, it is also unlikely that long-term chronic dehydration may be reversed by increased fluid intake in geriatric or long-term settings [33]. Disregarding all limitations of other symptoms and tests for water-loss dehydration in the aged, only 5 patients showed clinical signs diagnosed by a geriatrician.

According to our findings, use of diuretics seems to be related to dehydration (assessed with serum osmolality). Our findings support previous studies indicating that diuretic use can increase risk of developing dehydration $[34,35]$.

\section{Intraindividual Associations}

Highest correlations were measured between the skin surface $\mathrm{pH}$ of legs and arms or between frontal foreheads and arms. Skin surface $\mathrm{pH}$ seems to be a rather stable variable that was higher at legs, which is in accordance with the regional variability shown in earlier studies $[23,36]$.

Regarding the individual hydration parameters, results indicate that in geriatric patients the within-subject variability for the skin variables was smaller than the betweensubject variability. This pattern was supported recently [37] and may be useful for developing "skin tests" for water-loss dehydration or other phenomena of interest.

\section{Limitations}

A possible limitation of our study is the small sample size. Additionally, patients who had been diagnosed with heart failure, renal failure, or diabetes mellitus were also included in the study. This might have increased the serum osmolality, without an existing water-loss dehydration. Another limitation was the short-term monitoring of fluid intake, which did not allow us to assess the predictive value of fluid intake monitoring on dehydration in the longer term in older people. Because of the geriatric setting, fluid intake was highly controlled and showed a limited range. This may have decreased the chance to detect associations with other variables. The fluid intake was recorded by nurses, self-reported by the participants, and carefully extracted from the medical records. However, measurement errors may have occurred.

\section{Conclusions}

In a representative sample of geriatric patients, approximately half were diagnosed as being dehydrated according to widely accepted serum osmolality thresholds. At the same time, there was no association with fluid intake, 
questioning the clinical relevance of this measure. There is an urgent need to develop novel easy to use tests of dehydration in the aged, but empirical evidence supporting associations between skin barrier parameters and fluid intake and osmolality is weak. Especially epidermal hydration of the lower leg may play a role but most probably in combination with other tests. Further research is needed to provide scientific evidence for the question whether skin barrier parameters are associated with fluid intake and hydration status, especially in aged populations.

\section{Acknowledgements}

The authors wish to thank Gabor Dobos for contributing to study concept and design, the participants who completed the study, the managers and care staff of the Evangelische Geriatriezentrum Berlin (EGZB), where the study was completed.

\section{Statement of Ethics}

Participation was voluntary. Patients were only included after having signed the informed consent and the study conduct followed the principles of the Declaration of Helsinki.

\section{Disclosure Statement}

The authors declare that they have no conflicts of interest.

\section{Funding Sources}

This work was supported by the "Focus Area DynAge," the Clinical Research Center for Hair and Skin Science, Department of Dermatology and Allergy, Charité - Universitätsmedizin Berlin, and the Evangelische Geriatriezentrum Berlin (EGZB).

\section{References}

1 Popkin BM, D’Anci KE, Rosenberg IH: Water, hydration, and health. Nutr Rev 2010;68: 439-458.

-2 Thomas DR, Cote TR, Lawhorne L, Levenson SA, Rubenstein LZ, Smith DA, Stefanacci RG, Tangalos EG, Morley JE: Understanding clinical dehydration and its treatment. J Am Med Dir Assoc 2008;9:292-301.

- 3 Hooper L, Abdelhamid A, Attreed NJ, Campbell WW, Channell AM, Chassagne P, Culp KR, Fletcher SJ, Fortes MB, Fuller N, Gaspar PM, Gilbert DJ, Heathcote AC, Kafri MW, Kajii F, Lindner G, Mack GW, Mentes JC, Merlani P, Needham RA, Olde Rikkert MG, Perren A, Powers J, Ranson SC, Ritz P, Rowat AM, Sjostrand F, Smith AC, Stookey JJ, Stotts NA, Thomas DR, Vivanti A, Wakefield BJ, Waldreus N, Walsh NP, Ward S, Potter JF, Hunter P: Clinical symptoms, signs and tests for identification of impending and current water-loss dehydration in older people. Cochrane Database Syst Rev 2015;CD009647.

-4 Suhr JA, Hall J, Patterson SM, Niinisto RT: The relation of hydration status to cognitive performance in healthy older adults. Int J Psychophysiol 2004;53:121-125.

5 Secher M, Ritz P: Hydration and cognitive performance. J Nutr Health Aging 2012;16: 325-329.

6 Hooper L, Bunn D, Jimoh FO, FairweatherTait SJ: Water-loss dehydration and aging. Mech Ageing Dev 2014;136-137:50-58.

7 Megow I, Darvin ME, Meinke MC, Lademann J: A randomized controlled trial of green tea beverages on the in vivo radical scavenging activity in human skin. Skin Pharmacol Physiol 2017;30:225-233.
8 Jung S, Darvin ME, Chung HS, Jung B, Lee SH, Lenz K, Chung WS, Yu RX, Patzelt A, Lee BN, Sterry W, Lademann J: Antioxidants in Asian-Korean and Caucasian skin: the influence of nutrition and stress. Skin Pharmacol Physiol 2014;27:293-302.

\9 Palma L, Marques LT, Bujan J, Rodrigues LM: Dietary water affects human skin hydration and biomechanics. Clin Cosmet Investig Dermatol 2015;8:413-421.

10 Palma ML, Tavares L, Fluhr JW, Bujan MJ, Rodrigues LM: Positive impact of dietary water on in vivo epidermal water physiology. Skin Res Technol 2015;21:413-418.

11 Boelsma E, van de Vijver LP, Goldbohm RA, Klopping-Ketelaars IA, Hendriks HF, Roza L: Human skin condition and its associations with nutrient concentrations in serum and diet. Am J Clin Nutr 2003;77:348-355.

12 Wolf R, Wolf D, Rudikoff D, Parish LC: Nutrition and water: drinking eight glasses of water a day ensures proper skin hydration-myth or reality? Clin Dermatol 2010;28:380-383.

13 Folstein MF, Folstein SE, McHugh PR: "Minimental state." A practical method for grading the cognitive state of patients for the clinician. J Psychiatric Res 1975;12:189-198.

14 Mahoney FI, Barthel DW: Functional evaluation: The Barthel Index. Md State Med J 1965; 14:61-65.

15 Serup J: EEMCO guidance for the assessment of dry skin (xerosis) and ichthyosis: clinical scoring systems. Skin Res Technol 1995;1: 109-114.
16 du Plessis J, Stefaniak A, Eloff F, John S, Agner T, Chou TC, Nixon R, Steiner M, Franken A, Kudla I, Holness L: International guidelines for the in vivo assessment of skin properties in non-clinical settings. 2. Transepidermal water loss and skin hydration. Skin Res Technol 2013;19:265-278.

17 Fluhr JW, Feingold KR, Elias PM: Transepidermal water loss reflects permeability barrier status: validation in human and rodent in vivo and ex vivo models. Exp Dermatol 2006;15: 483-492.

18 Clarys P, Clijsen R, Taeymans J, Barel AO: Hydration measurements of the stratum corneum: comparison between the capacitance method (digital version of the Corneometer CM $825^{\circledR}$ ) and the impedance method (Skicon-200EX ${ }^{\circledR}$ ). Skin Res Technol 2012;18: 316-323.

19 Stefaniak AB, du Plessis J, John SM, Eloff F, Agner T, Chou T-C, Nixon R, Steiner MFC, Kudla I, Holness DL: International guidelines for the in vivo assessment of skin properties in non-clinical settings: part 1. pH. Skin Res Technol 2013;19:59-68.

20 Makrantonaki E, Steinhagen-Thiessen E, Nieczaj R, Zouboulis CC, Eckardt R: Prevalence of skin diseases in hospitalized geriatric patients: association with gender, duration of hospitalization and geriatric assessment. Z Gerontol Geriatr 2017;50:524-531.

21 Hahnel E, Blume-Peytavi U, Trojahn C, Dobos G, Stroux A, Garcia Bartels N, Jahnke I, Lichterfeld-Kottner A, Neels-Herzmann $\mathrm{H}$, Klasen A, Kottner J: The effectiveness of standardized skin care regimens on skin dryness in nursing home residents: a randomized controlled parallel-group pragmatic trial. Int J Nurs Stud 2017;70:1-10. 
22 Mentes J: Oral hydration in older adults: greater awareness is needed in preventing, recognizing, and treating dehydration. Am J Nurs 2006;106:40-49; quiz 50.

-23 Zlotogorski A: Distribution of skin surface $\mathrm{pH}$ on the forehead and cheek of adults. Arch Dermatol Res 1987;279:398-401.

24 Sato N, Kitahara T, Fujimura T: Age-related changes of stratum corneum functions of skin on the trunk and the limbs. Skin Pharmacol Physiol 2014;27:181-181.

25 Blaak J, Kaup O, Hoppe W, Baron-Ruppert G, Langheim H, Staib P, Wohlfart R, Lüttje D, Schürer N: A long-term study to evaluate acidic skin care treatment in nursing home residents: impact on epidermal barrier function and microflora in aged skin. Skin Pharmacol Physiol 2015;28:269-279.

26 Lichterfeld A, Lahmann N, Blume-Peytavi U, Kottner J: Dry skin in nursing care receivers: A multi-centre cross-sectional prevalence study in hospitals and nursing homes. Int J Nurs Stud 2016;56:37-44.

27 Palma ML, Monteiro C, Tavares L, Bujan MJ, Monteiro Rodrigues L: Relationship between the dietary intake of water and skin hydration. Biomed Biopharm Res 2012;9:173-181.
28 Eisenbeiss C, Welzel J, Eichler W, Klotz K: Influence of body water distribution on skin thickness: measurements using high-frequency ultrasound. Br J Dermatol 2001;144. 947-951.

29 Roh NK, Kim MJ, Lee YW, Choe YB, Ahn KJ: A split-face study of the effects of a stabilized hyaluronic acid-based gel of nonanimal origin for facial skin rejuvenation using a stamptype multineedle injector: a randomized clinical trial. Plastic Reconstr Surg 2016;137:809816.

30 Williams S, Krueger N, Davids M, Kraus D, Kerscher M: Effect of fluid intake on skin physiology: distinct differences between drinking mineral water and tap water. Int J Cosmet Sci 2007;29:131-138.

-31 Smith DR, Atkinson R, Tang S, Yamagata Z: A survey of skin disease among patients in an Australian nursing home. J Epidemiol 2002; 12:336-340.
32 National Clinical Guideline Centre (UK): Patient Experience in Adult NHS Services: Improving the Experience of Care for People Using Adult NHS Services: Patient Experience in Generic Terms. London, Royal College of Physicians, National Clinical Guideline Centre, 2012.

33 Massler M: Geriatric nutrition II: dehydration in the elderly. J Prosthet Dent 1979;42:489491.

34 Hooper L, Bunn DK, Downing A, Jimoh FO, Groves J, Free C, Cowap V, Potter JF, Hunter PR, Shepstone L: Which frail older people are dehydrated? The UK DRIE Study. J Gerontol A Biol Sci Med Sci 2016;71:1341-1347.

35 Lancaster KJ, Smiciklas-Wright H, Heller DA, Ahern FM, Jensen G: Dehydration in black and white older adults using diuretics. Ann Epidemiol 2003;13:525-529.

$>36$ Wilhelm KP, Cua AB, Maibach HI: Skin aging. Effect on transepidermal water loss, stratum corneum hydration, skin surface $\mathrm{pH}$, and casual sebum content. Arch Dermatol 1991; 127:1806-1809.

37 Hahnel E, Blume-Peytavi U, Trojahn C, Kottner J: Associations between skin barrier characteristics, skin conditions and health of aged nursing home residents: a multi-center prevalence and correlational study. BMC Geriatr 2017;17:263. 